\title{
Effect of DC poling duration on space charge relaxation in virgin XLPE cable peelings
}

\author{
Antonios Tzimas ${ }^{1}$, Simon M Rowland ${ }^{1}$, Leonard A Dissado ${ }^{2}$, Mingli Fu ${ }^{3}$ and Ulf \\ H Nilsson ${ }^{4}$ \\ ${ }^{1}$ University of Manchester, School of Electrical and Electronic Engineering, \\ Manchester, M60 1QD, UK. \\ ${ }^{2}$ University of Leicester, Department of Engineering, Leicester, LE1 7RH, UK. \\ ${ }^{3}$ AREVA T\&D UK Limited, St Leonards Avenue, Stafford, ST17 4LX, UK. \\ ${ }^{4}$ Borealis AB, SE-444 86, Stenungsund, Sweden. \\ Email: Antonios.Tzimas@manchester.ac.uk
}

\begin{abstract}
The effect of DC poling time upon the time-dependent decay of space charge in insulation peelings of Cross-linked Polyethylene (XLPE) cable that had not previously experienced either electrical or thermal stressing is investigated. Two DC poling durations were used, two hours and twenty six hours at an electric field of $50 \mathrm{kV} / \mathrm{mm}$ and at ambient temperature. Space charge was measured in the two samples investigated both during space charge accumulation and throughout its subsequent decay. The results show that the length of DC poling plays an important role in the subsequent decay. Despite the fact that both samples have had the same amount of space charge by the end of both short and long poling durations the time dependence of the space charge decay is different. Most of the charge stored in the sample that had experienced the short time poling decays rapidly after voltage removal. On the other hand the charge that is stored in the sample with the long DC poling duration decays slowly and its decay occurs in two stages. The data, which is analysed by means of the detrapping theory of space charge decay, implies that the charge stored in the material has occupied energy states with different trap depth ranges. The two poling durations lead to different relative amounts of charge in each of the two trap depth ranges. Possible reasons for this are discussed.
\end{abstract}

\section{Introduction}

It has been suggested that space charge built up in High Voltage (HV) cable insulation is either the cause [1] of material degradation or a consequence of it [2], with subsequent electrical tree formation eventually leading to catastrophic failure of the insulation. Many studies [3-7] as well as an EUsponsored programme called ARTEMIS [8] have shown that quantities that can be extracted from space charge measurement are sensitive to polymeric material changes due to electrical, thermal or electro-thermal stressing [9]. Studies of space charge behaviour on cable peelings have shown to be sensitive to prior cable stressing [10] and/or further endurance stressing [11-13]. Nevertheless, the role of space charge in the degradation and subsequent ageing of the polymeric insulation materials is still not well understood. The dynamics of space charge formation and removal can be followed by a 
number of measuring techniques such as the Pulsed-Electro-Acoustic (PEA) technique [14] used here. However the interpretation of the resulting data presents a challenge since space charge by definition is the net charge in a given locality. This results from the accumulation of charges of both positive and negative polarity whose dynamics are separately governed by a number of processes such as mobility, trapping and de-trapping, ionization and recombination as well as injection and extraction all of which need to be considered in the formulation of a model $[15,16,17]$. This is especially the case during space charge accumulation when the relative importance of the various processes may vary in time as the field and other factors change. The aim of this work is to elucidate the effect of DC poling duration upon the trap distribution of space charge, by analyzing the charge de-trapping characteristics of the subsequent relaxation dynamics. Peelings taken from virgin cross-linked polyethylene (XLPE) cable are used to avoid any effects of prior stress history. The analysis of the space charge decay measurements is carried out by examining the decay characteristics of the total charge stored in each sample using the theoretical model proposed in [18].

\section{Experimental}

\subsection{Sample fabrication and handling}

The virgin cable peelings that are used in this study are a product of a European funded programme called ARTEMIS [8]. The peelings have a thickness of $150 \mu \mathrm{m}$ and were peeled with a specially designed lathe to ensure uniformity of the thickness and surface roughness [19]. One material supplier provided two cable manufactures with the same batch of XLPE resin in order to make model cables for Extra High-Voltage (EHV) with an insulation thickness of $14 \mathrm{~mm}$. This corresponds to a $90 \mathrm{kV}$ cable construction, as shown in Figure 1a. The peelings were rolled into tapes with a width of $8 \mathrm{~cm}$ as is shown in Figure 1b and were distributed to the rest of the ARTEMIS partners inside polyethylenebags completely enclosed within aluminium bags. From then onwards the tapes were stored at a temperature around $278 \mathrm{~K}$ in order to prevent any further changes. The properties of the peelings were then investigated using samples taken from between $2 \mathrm{~mm}$ to $4 \mathrm{~mm}$ (or as close as possible within those margins) from the inner semicon screen. Before carrying out any space charge measurements the samples were conditioned [19] at $323 \mathrm{~K}$ for 48 hours at ambient pressure so as to remove any volatile chemicals that are known to influence the behaviour of space charge in an undefined manner [8]. The experiments reported here were carried out on two samples cut from the same peeling taken from a cable that had not been placed under any kind of stress i.e. it was unaged when the peeling was taken. In order to differentiate the two samples from the peeling of the virgin cable that were also examined we will refer to them as UA2h and UA26h. The acronyms UA stand for Un-Aged material and the " $2 \mathrm{~h}$ " and " $26 \mathrm{~h}$ " refer to the poling duration of the samples. Only one sample was used for each of the poling durations, and hence the results reported are sample-specific and not averaged values. However previous work $[9,10]$ has confirmed the same pattern of behaviour in other samples of the same type.

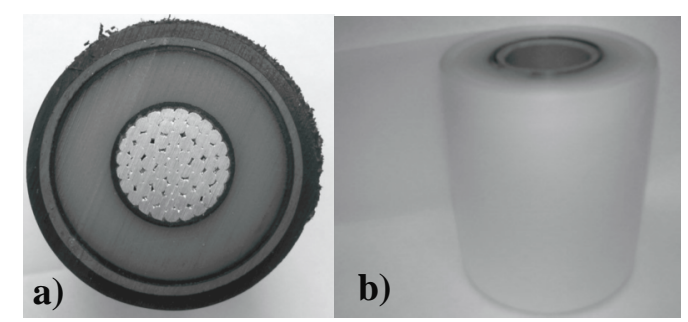

Figure 1. a) Section of the cable after any kind of stressing during the ARTEMIS programme and b) a tape roll after the insulation was peeled.

\subsection{Pulsed-Electro-Acoustic [14] technique protocol}

The HV electrode is a disc of semicon material with a radius of $75 \mathrm{~mm}$ and the ground electrode aluminium. A drop of silicone oil was placed between the electrodes and the sample to ensure good 
acoustic propagation. A positive voltage of $7.5 \mathrm{kV}$, i.e. a DC poling field of $50 \mathrm{kV} / \mathrm{mm}$, was applied at ambient temperature for either 2 hours or 26 hours. During the poling time a series of measurements were made in which the voltage was switched off transiently so that the space charge and corresponding image charges on the electrodes could be measured without the overlay of the capacitive charge. The space charge profile measurement data was processed using the method described in [20], modified for flat samples. The voltage was switched off at the end of the poling period and the decay of the space charge monitored at regular intervals so as to obtain the space charge decay dynamics.

\section{Results}

An initial measurement was carried out using two semicon electrodes and no oil. Figure 2 shows the space charge distribution $2 \mathrm{~min}$ and $14 \mathrm{~min}$ after voltage removal subsequent to two hours of poling. It can be seen that the negative space charge peak next to the cathode electrode has substantially the same magnitude as that found subsequently using the silicone oil (Figure 3a), but the anode-electrode peak was very broad and difficult to de-convolute compared to that obtained using the silicone oil. All our other measurements were therefore obtained using silicone oil to maintain an acoustic contact.

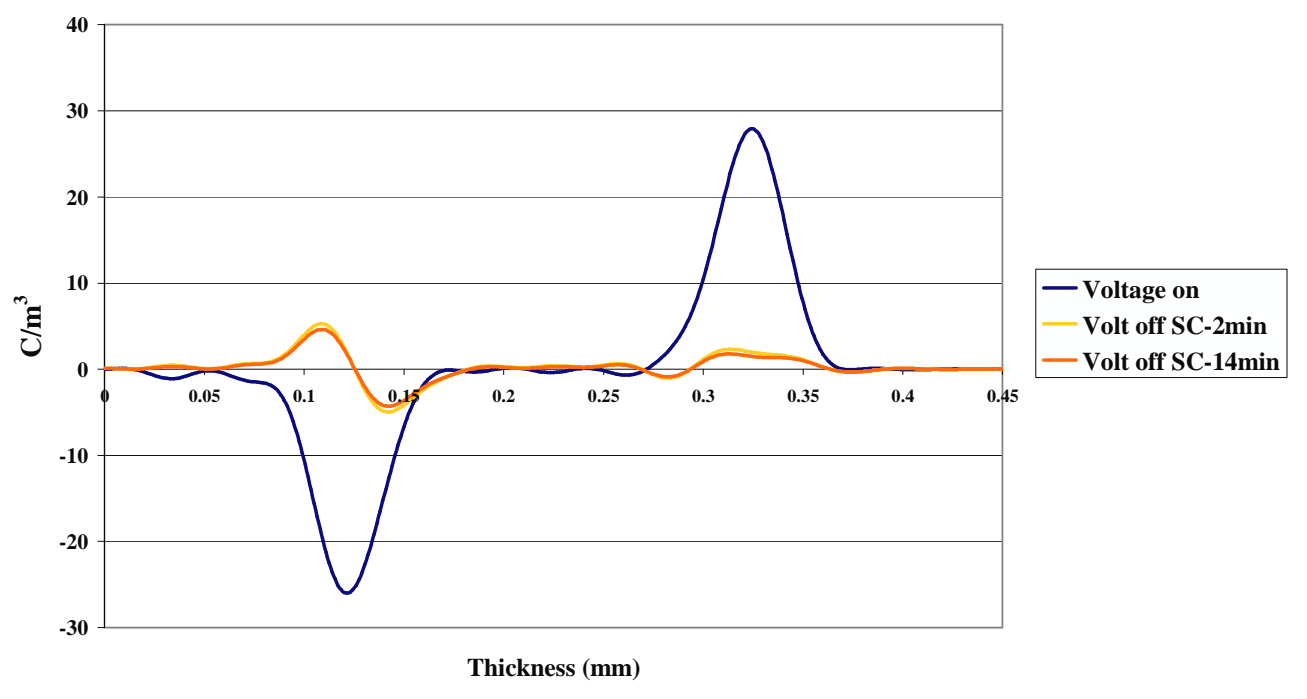

Figure 2. Space Charge distribution for a sample measured between semicon electrodes without silicone oil.

Figure 3 contrasts the space charge distribution, and its decay following voltage removal, observed in the samples after two hours poling (UA2h, Fig. 3a) with that observed after twenty six hours of poling (UA26h, Fig. 3b). Both samples contain a negative space charge peak next to the electrode that served as a cathode during poling, however the sample poled for twenty six hours also contained a negative peak next to the electrode that was the anode during poling. The two positive peaks are the induced charge on the electrodes, with the small negative dip in the response from the ex-anode electrode attributed to the shape of the instrument response to the probe pulse. As it should be, the sum of space charge and induced charge is close to zero in all measurements except the first one where the peak on the ex-anode electrode is anomalously large. The data from the first measurement, estimated to correspond to a decay time of six seconds, must therefore be treated with some caution. The measurements therefore imply the injection of negative charge from the cathode, which remains trapped close to the injecting electrode when the poling duration is two hours. When the poling duration is extended to twenty six hours however some of the injected charge is transported to the anode where it becomes trapped as a negative heterocharge region. In addition the decay of the space charge in the sample poled for twenty six hours is much slower than in the one poled for only two 
hours. This is particularly marked in the case of the heterocharge peak next to the ex-anode electrode, but is also true of the negative homocharge peak. The subsequent sections analyse the reason for this difference in terms of charge trap distributions and comment on the implications of the analysis for the interpretation of space charge dynamics.
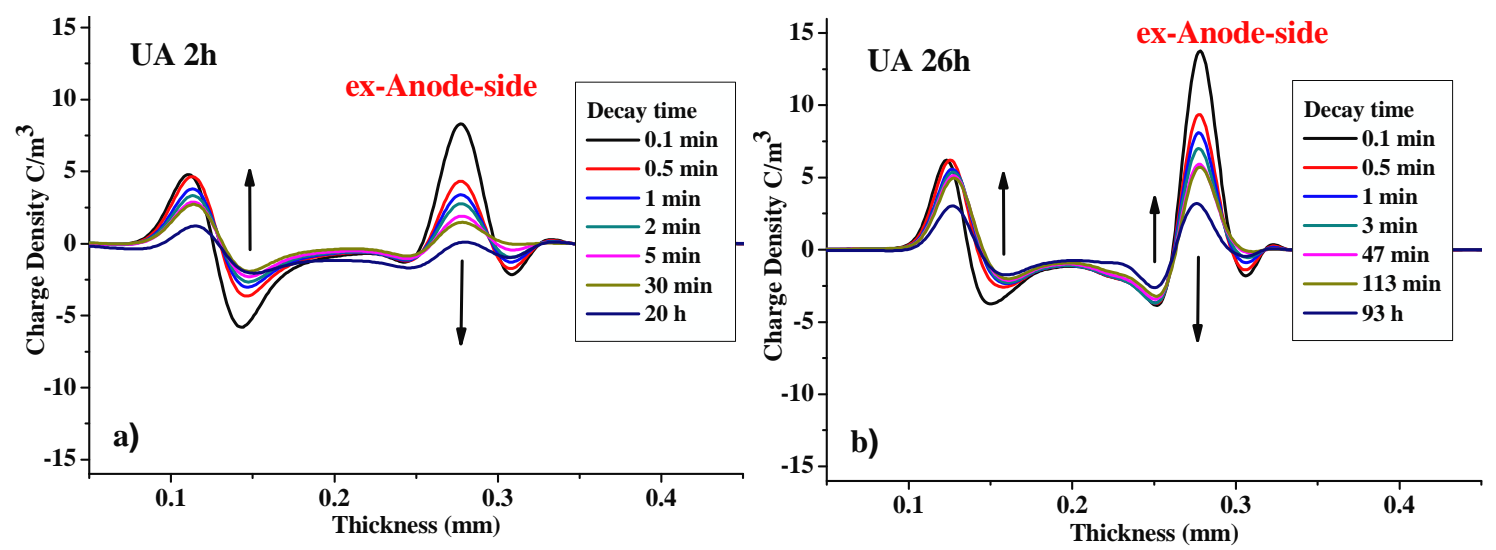

Figure 3. The space charge decay profiles for short time (two-hour) DC poling (a), and long time (26-hour) DC poling (b).

\section{Theoretical model}

The neutralization of space charge following voltage removal may involve one or more of a number of mechanisms. Neutralization by Schottky injection usually involves the injection of negative charges [21,22], although an example of neutralization by both negative and positive charge is given in [23], and takes place when the space charge field at the neutralizing electrode is high [21-23]. In the case of epoxy resin [22] this mechanism becomes negligible after ten seconds and the residual space charge is removed much more slowly. Two models have been presented for the slow removal of space charge. In one the rate determining step is the de-trapping of the charge from the trap states, with the transport of the charge to the electrodes for neutralization being a negligible fraction of the total decay time [18, 24]. This model is consistent with the usual interpretation of Thermally Stimulated Discharge Currents (TSDC) [25, 26]. An alternative model [3] is based on the transport rate of charge carriers in a system containing decaying space charge. A number of approximations are used to relate the changing space charge field to the time dependent space charge density and hence determine an effective mobility for the charge extracted at a given time. This mobility reduces in time as the depth of the lowest empty traps reduces due to the removal of the space charge. Thus despite their different mathematic expression these two models contain the same physical concepts, namely that the removal of space charge at a given time is determined by the highest trap level remaining filled at that time.

As it contains less approximations and its relationship to the trap filling is more transparent we shall use the de-trapping model $[18,24]$ to analyse the data. Details of this model are given in Appendix 1 of [18] so here we shall confine ourselves to a brief outline of the physics behind its quantitative formulation. The starting point is the assumption that the space charge resides in traps with an energy distribution that is taken to be uniform over a given range of trap depths, i.e. it has a constant number of traps per unit energy per cubic metre $(N)$ over this range. This form of distribution relates to traps of a specific type whose trap energy is broadened by the various local environments in the polymer, and is chosen here because it is a first approximation to the peaks observed in (TSDC) measurements [26]. At $t=0$ these traps are taken to be fully filled up from the deepest trap in this population at a trap depth of $\Delta_{\max }$ up to traps at a depth of $\Delta_{\min }$, which is not necessarily the extent of 
the trap depth range of the population but rather the highest filled trap depth. For a single population of traps $\Delta_{\min }$ is therefore the demarcation level or pseudo-Fermi energy [27].

The release rate of charges from traps at a depth $\Delta$ is taken to be independent of release of charge from traps at different depths and thus the rate of change of the concentration of filled traps at $\Delta$ becomes,

$d N(t) / d t=-N(t) \operatorname{vexp}(-\Delta / \mathrm{kT})$

Here $v$ is the attempt frequency for release of the carrier and k is Boltzmann's constant. Since $N(t) \mathrm{d} \Delta$ is the concentration (number per cubic metre) of filled traps with trap depths between $\Delta$ and $\Delta+\mathrm{d} \Delta$, integration of Equation (1) over the filled trap range $\left(\Delta_{\max }\right.$ to $\left.\Delta_{\min }\right)$ and multiplication by the electron charge e gives the rate of change of the space charge density $\rho(t)$ as,

$\mathrm{d}\{\rho(\mathrm{t})\} / \mathrm{dt}=(\mathrm{eNkT/t})\left[\exp \left\{-v \mathrm{t} \mathrm{Y}_{\max }\right\}-\exp \left\{-v \mathrm{t} \mathrm{Y}_{\min }\right\}\right]$

with

$\mathrm{Y}_{\max }=\exp \left(-\Delta_{\min } / \mathrm{kT}\right)$ and $\mathrm{Y}_{\min }=\exp \left(-\Delta_{\max } / \mathrm{kT}\right)$

Integrating Equation (2) between $t$ and infinity gives the space charge density remaining at time $t$ as,

$\rho(\mathrm{t})=\mathrm{e} N \mathrm{kT}\left[\mathrm{E}_{1}\left(v \mathrm{t} \mathrm{Y}_{\min }\right)-\mathrm{E}_{1}\left(v \mathrm{t} \mathrm{Y}_{\max }\right)\right] \equiv \mathrm{Q}(\mathrm{t}) /$ Volume

In Equation (4) $E_{1}(x)$ is the exponential integral [28] defined by

$$
E_{1}(x)=\int_{x}^{\infty} \frac{\exp (-t)}{t} d t
$$

Equation (4) for the charge density can be converted to an equation for the time dependence of the total space charge (Q) by integrating it over the spatial distribution of $\boldsymbol{N}$. Under the assumption that the trap distribution is spatially uniform this is equivalent to replacing $N$ by $\mathrm{N}(=N \mathrm{x}$ Volume) the total number of traps per unit energy. The resulting time dependence of $\mathrm{Q}$ is determined by the functions $\mathrm{E}_{1}\left(v t \mathrm{Y}_{\min }\right)$ and $\mathrm{E}_{1}\left(v t \mathrm{Y}_{\max }\right)$ and is illustrated in Figure 4a. The shape of this plot can be understood by reference to the asymptotic expressions for $\mathrm{E}_{1}(\mathrm{x})$,

$$
\begin{aligned}
& E_{1}(x) \approx-\ln (x)-0.57721 \quad \mathrm{x}<1 \\
& E_{1}(x) \approx \frac{\exp (-x)}{x} \quad \mathrm{x}>1
\end{aligned}
$$

Denoting $\left(v Y_{\max }\right)^{-1}$ as $t_{1}$ and $\left(v Y_{\min }\right)^{-1}$ as $t_{2}$ it can be seen that when the time $t<<t_{1}, t_{2}$ the time dependence from the logarithmic terms of Equation (6) cancel and $\mathrm{Q}$ approaches its initial $(\mathrm{t}=0)$ value of

$$
\mathrm{Q}(0)=\mathrm{eN}\left[\Delta_{\max }-\Delta_{\min }\right]
$$




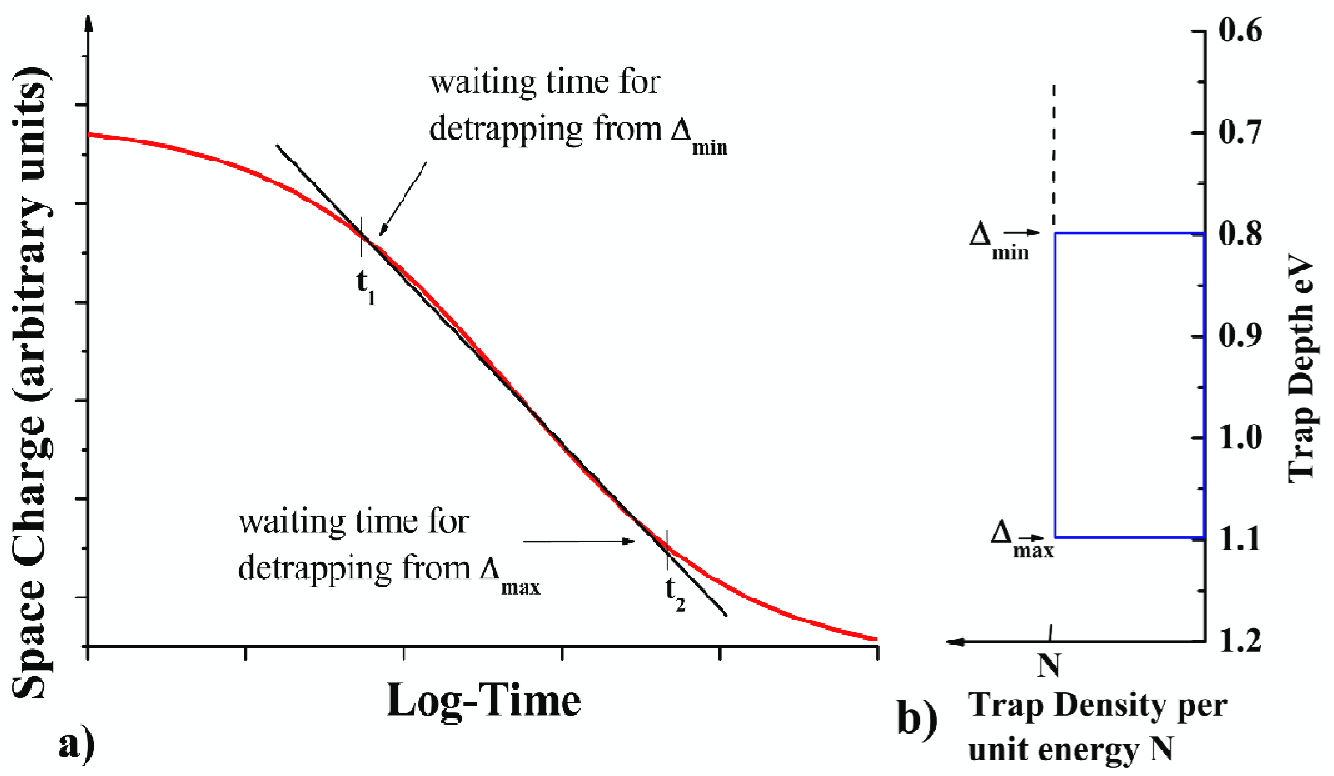

Figure 4. (a) Diagram illustrating the time dependence of space charge decay predicted by Equation (4) [18], (b) diagram of filled charge traps corresponding to the decay illustrated in (a).

The exact series expressions for $E_{1}(x)$ tabulated in [28] give a small decrease over time until $t=t_{1}$ after which $\mathrm{E}_{1}\left(v t \mathrm{Y}_{\min }\right)$ approaches the small values of the large $\mathrm{x}$ asymptotic limit leaving the logarithmic term of $E_{1}\left(v t Y_{\max }\right)$ as the only significant contribution. This time $t_{1}$ corresponds to the time at which a significant amount of charge is released from the highest filled trap state with trap depth $\Delta_{\min }$. The region where $\mathrm{Q}(\mathrm{t})$ is linearly dependent upon $\log ($ time $)$ corresponds to the emptying of traps of progressively larger trap depth and continues until $t=t_{2}$ at which time the traps being emptied correspond to the deepest filled trap states at a depth of $\Delta_{\max }$. Thus,

$$
\mathrm{Q}(\mathrm{t}) \approx \mathrm{a}-\mathrm{Q}(0) \mathrm{kT} /\left[\Delta_{\max }-\Delta_{\min }\right] \ln (\mathrm{t})=\mathrm{a}-\operatorname{eNkTln}(\mathrm{t}) \quad \exp \left(\frac{\Delta_{\min }}{k T}\right)<v t<\exp \left(\frac{\Delta_{\max }}{k T}\right)
$$

Here "a" is a constant. When $t>t_{2}$ the value of $Q(t)$ reduces slowly to zero as the deepest filled trap states are emptied. A plot of the measured space charge as a function of $\log (\mathrm{t})$ would therefore allow the trap depth range and trap concentration to be determined. The parameters can be deduced from the time $t_{1}$ and $t_{2}$ at the beginning and end of the logarithmic region, the asymptotic zero time value of $\mathrm{Q}(\mathrm{t})$, and the gradient of the logarithmic portion given by eNkT $\left(=\mathrm{Q}(0) \mathrm{kT} /\left[\Delta_{\max }-\Delta_{\min }\right]\right)$. The times can be used to deduce the trap depths $\Delta_{\min }$ and $\Delta_{\max }$, providing that, $\mathrm{v}$, the de-trapping attempt frequency can be estimated. We have taken $v=\mathrm{kT} / \mathrm{h}$ for our estimation as this frequency relates to incoherent thermal vibrations of the trap site. An exact value for $v$ could be obtained from measurements at a range of temperatures. Where one or other of these times are unavailable, measurement of $\mathrm{Q}(0)$ and the gradient of the logarithmic portion can be used to make an estimate of the trap depth corresponding to the unmeasured time. Similarly measurement of the gradient and the two times will yield an estimate for $\mathrm{Q}(0)$. Figure $4 \mathrm{~b}$ is an illustration of the filled trap energy distribution corresponding to a decay plot such as that shown in Figure 4a. The trap depths denoted cover a typical range observed in experiments $[18,24]$ and correspond to times $t_{1}=4 \mathrm{~s}$ and $t_{2}=$ $4.76 \times 10^{5} \mathrm{~s}$ at $\mathrm{T}=300 \mathrm{~K}$ when $v=\mathrm{kT} / \mathrm{h}$. 


\section{Space Charge Decay: Results and Analysis}

It is not always clear with space charge measurements where the bulk space charge ends and the induced charge on the electrode begins so in the first instance we have calculated the total absolute charge in the charge distribution using Equation (10).

$Q_{t o t}(t)=A \int_{x_{1}}^{x_{o}}|\rho(x, t)| d x$

Here $A$ is the surface area of the HV electrode, $\rho(x, t)$ is the space charge density, and $t$ is the time at which the measurement is carried out after removal of the voltage. The integral is taken between positions $x_{0}$ and $x_{1}$, which lie outside of the measured space charge region, and thus includes the image charges on the electrodes. Since the net charge should be zero in the absence of an applied potential the induced charge on the electrodes should be equal in magnitude and opposite in polarity to the bulk space charge, hence division of this value by two gives the absolute value of the space charge $(\mathrm{Q}(\mathrm{t}))$ stored in the bulk of the material,

$\mathrm{Q}(\mathrm{t})=(1 / 2) \mathrm{Q}_{\mathrm{tot}}(\mathrm{t})$

These values of $Q(t)$ are plotted as a function of $\log (t)$ in Figure 5a, for both the samples poled for two hours and the one poled for twenty six hours. Although the earliest data point should be regarded with some caution it is noticeable that its value is consistent with the later points and hence it is retained in the analysis. The figure shows that the space charge decay involves two time regimes, a short time decay that ceases around $10^{3} \mathrm{~s}$ and is dominant in the UA2h sample but also occurs in the UA26h sample and a long term decay that starts around a few thousand seconds and goes on beyond the timescale of the experiment $\left(\sim 3 \times 10^{5} \mathrm{~s}\right)$. This latter regime is particularly prominent in the UA26h sample. Since Equation (10) gives a space charge measure that does not distinguish between the homocharge negative peak at the cathode side and the negative heterocharge peak at the anode side it is possible that the two regimes relate to the two different peaks, although previous data from epoxy resin where only one peak was involved show a similar behaviour. This point will be investigated later in the paper.
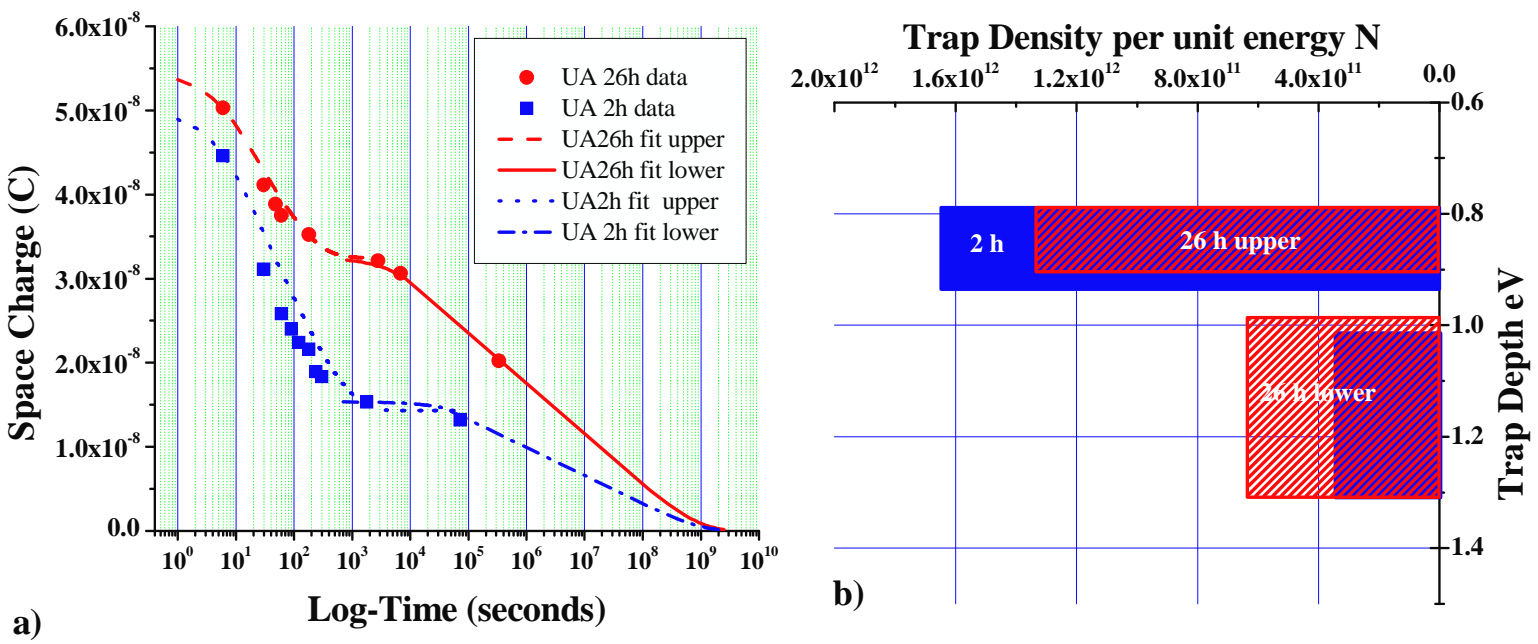

Figure 5. (a) Space charge decay of UA after 2 and 26 hour of poling, calculated using Equation (10), (b) trap depth distribution of space charge after 2 (in solid filling) and 26 (in stripes) hours. 
A comparison of the shape of the plots in Figure 5a with the decay model in Figure 4a suggests that we should interpret the data as being due to the superposition of the release of charge from two different populations of traps with different ranges of trap depths. One population is responsible for the short term decay, which is prominent in both the UA2h and the UA26h samples. The other population lying to lower energies is responsible for the long term decay and is prominent in the UA26h sample. Although the long term decay is not so well established in the UA2h sample its existence is clear from the plateau of $\sim 1.5 \times 10^{-8} \mathrm{C}$ in the residual space charge at times between $\sim 2 \times 10^{3} \mathrm{~s}$ and $8 \times 10^{4} \mathrm{~s}$. The space charge decay data is therefore fitted to the sum of charge release from two trap populations each one described separately by Equation (4). The temperature used in the fitting is taken to be $300 \mathrm{~K}$. First estimates of the parameters are obtained from the shape of the curve as described in the previous section. The space charge plateau around $10^{3} \mathrm{~s}$ marks the cross-over between release from the shallow traps and release from the deep traps, and gives a first estimate to the $Q(0)$ value for the deep trap population. The times $t_{2}$ for the shallow traps and $t_{1}$ for the deep traps can be obtained directly from the finish and start of regions where $Q(t)$ is proportional to $\log (t)$, and the gradient in the regions where the data is proportional to $\log (\mathrm{t})$ yields estimates of $\Delta_{\min }$ for the shallower trap population and $\Delta_{\max }$ for the deeper trap population as $\mathrm{t}_{1}$ appropriate to the former case and $t_{2}$ appropriate to the latter case do not fall within our time range. The first estimates are then adjusted to obtain the best fit over the whole time range. This is shown by the dotted line for UA2h and by the dashed line for UA26h in Figure 5a. The other lines show the fit to just the long term decay from the deep traps. Figure $5 \mathrm{~b}$ shows the two ranges of filled traps corresponding to the fits plotted in Figure 5a, from which it appears that there is a gap in energy between the two filled trap ranges. This does not necessarily imply that there are no trap states in this energy region just that any that exist must have a small population, which would imply a small curvature in the plateau region where the short term and long term decay cross that the detail in our measurements is insufficient to reveal.

Figure 5a indicates that the amount of space charge stored in total, i.e. the sum of that stored in each trap population is almost the same for both UA2h and UA26h, but the rates of decay are very different. The parameters used in the fitting are given in Table 1 and lead to the same conclusion. The decay slope, in the $\log (\mathrm{t})$ plot, of the $2 \mathrm{~h}$-poled sample is very steep, thus $70 \%$ of the charge has escaped/neutralized within 860 seconds. On the other hand the decay slope of the $26 \mathrm{~h}$-poled sample has two clear-cut regions one corresponding to the release from shallow traps, which is of a similar decay rate to that of UA2, and a much slower one of release from the deeper traps that now are populated by a much higher fraction of the total charge stored. The parameters in Table 1 show that the space charge that decays during the first few hundred seconds corresponding to the upper part of the slope was trapped in energy states with trap depths in the range between 0.81 and $0.91 \mathrm{eV}$, with $1.34 \times 10^{12}$ traps per $\mathrm{eV}$ in the sample volume measured. The space charge that decays at longer time corresponding to the lower part of the slope was trapped in states with a trap depth range between 0.99 and $1.3 \mathrm{eV}$, with $6.43 \times 10^{11}$ traps per $\mathrm{eV}$. On the other hand most of the space charge in the $2 \mathrm{~h}$-poled sample was trapped in energy states in the trap depth range between 0.81 and $0.94 \mathrm{eV}$ with $1.62 \times 10^{12}$ traps per $\mathrm{eV}$ for the upper part of the slope. However by the end of the last decay measurement of the $2 \mathrm{~h}$-poled sample there is still some charge retained that escapes slowly. Hence, in order to find out the number of energy states that are occupied, it is assumed that the maximum trap depth of the short time poling sample, $\Delta_{\max }$, is the same as that estimated for the 26h-poled sample. This is reasonable as both samples are cut from the same location in the same tape. In this way it is found that the charge retained for longer time resides in energy states in the trap depth range between 1.05 and $1.3 \mathrm{eV}$, with $3.75 \times 10^{11}$ traps per $\mathrm{eV}$. When the number of occupied energy states per energy interval $(\mathrm{eV})$ corresponding to the upper and lower part of the plot the UA26h sample is added together it is found that the total amount is almost equal to the equivalent total amount of traps per eV occupied in the UA2h, see Equation (12). 
Table 1. Parameters derived for the shallow and deep trap distributions from the decay of the charge stored after 2 and 26 hours of poling of unaged (UA) material.

\begin{tabular}{|c|c|c|c|c|}
\hline Parameters & $\begin{array}{l}\text { UA } 2 \mathrm{~h} \text { of poling } \\
\text { (shallow traps) }\end{array}$ & $\begin{array}{l}\text { UA } 2 \mathrm{~h} \text { of poling } \\
\text { (deep traps) }\end{array}$ & $\begin{array}{c}\text { UA } 26 \mathrm{~h} \text { of } \\
\text { poling } \\
\text { (shallow traps) }\end{array}$ & $\begin{array}{l}\text { UA 26h of poling } \\
\text { (deep traps) }\end{array}$ \\
\hline $\begin{array}{c}\mathrm{Q}(0)=\mathrm{eN}\left[\Delta_{\max }-\right. \\
\left.\Delta_{\min }\right]\left(\text { Coulombs) }{ }^{\mathrm{a}}\right.\end{array}$ & $3.465 \times 10^{-8}$ & $1.53 \times 10^{-8}$ & $2.2 \times 10^{-8}$ & $3.25 \times 10^{-8}$ \\
\hline $\mathrm{t}_{1}$-seconds & 6 & 69786 & 6 & 6780 \\
\hline$\Delta_{\min }(\mathrm{eV})$ & 0.81 & 1.05 & 0.81 & 0.99 \\
\hline$\Delta_{\max }(\mathrm{eV})$ & 0.94 & 1.30 & 0.91 & 1.30 \\
\hline $\mathrm{Q}(0) \mathrm{kT} /\left[\Delta_{\max }-\Delta_{\min }\right]$ & $6.72 \times 10^{-9}$ & $1.55 \times 10^{-9}$ & $5.55 \times 10^{-9}$ & $2.66 \times 10^{-9}$ \\
\hline $\mathrm{t}_{2}$-seconds & 1214 & $1.32 \times 10^{9}$ & 287 & $1.32 \times 10^{9}$ \\
\hline $\begin{array}{l}\text { N-number of energy } \\
\text { states in stressed } \\
\text { volume per eV } \\
\text { energy interval. }\end{array}$ & $\begin{array}{c}1.62 \times 10^{12} \\
\left(6.12 \times 10^{19}\right)^{b}\end{array}$ & $\begin{array}{c}3.75 \times 10^{11} \\
\left(1.41 \times 10^{19}\right)^{b}\end{array}$ & $\begin{array}{c}1.34 \times 10^{12} \\
\left(5.06 \times 10^{19}\right)^{b}\end{array}$ & $\begin{array}{c}6.43 \times 10^{11} \\
\left(2.42 \times 10^{19}\right)^{b}\end{array}$ \\
\hline $\mathrm{N}_{\text {Total }}{ }^{\mathrm{c}}$ & \multicolumn{2}{|c|}{$1.99 \times 10^{12}$} & \multicolumn{2}{|c|}{$1.98 \times 10^{12}$} \\
\hline
\end{tabular}

${ }^{\mathrm{a}}$ The total charge is obtained by summing the charge stored in the deep and shallow traps.

${ }^{\mathrm{b}}$ The concentration of filled traps per $\mathrm{eV}\left(\mathrm{m}^{-3}(\mathrm{eV})^{-1}\right)$ is shown in brackets.

${ }^{\mathrm{c}}$ Total number of filled energy states per eV energy interval.

$N_{\text {UA26h(upper })}+N_{\text {UA26h(lower })} \approx N_{U A 2 h \text { (upper })}+N_{U A 2 h(\text { lower })}$

$N_{U A 26 h(\text { Total })} \approx N_{U A 2 h(\text { Total })}$

This means that the total amount of charge that is occupying the trap states after dc poling of 2 and 26 hours is very similar, as the overall range of trap depths is similar. However in the case of 2 hours poling the larger amount of space charge is residing in the energy states with smaller de-trapping energies, i.e. shallow traps, whereas in the case of 26 hours poling the space charge mostly resides in energy states with larger de-trapping energies, i.e. deep traps. It therefore seems that the system has not reached thermal equilibrium within 2 hours of DC poling, i.e. the injected charge carriers have not filled all the available deep traps, and has a population in the shallow traps that does not yet obey the Boltzmann (or better Fermi) distribution giving the population of electronic carriers in a state at energy $E_{i}$ as,

$$
N_{i}\left(1+\exp \left(\frac{\left(E_{i}-E_{F}\right)}{k T}\right)\right)^{-1}
$$

Here $\mathrm{N}_{\mathrm{i}}$ is the density of trap states at energy $\mathrm{E}_{\mathrm{i}}$ and $\mathrm{E}_{\mathrm{F}}$ is the Fermi energy. 


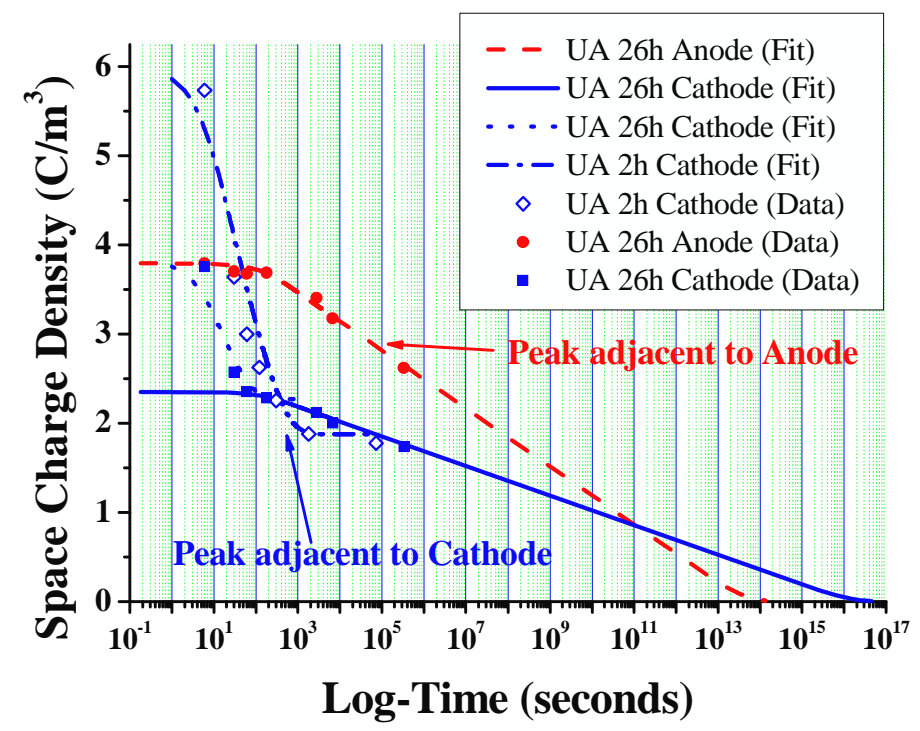

Figure 6. Decay of the space charge density stored at the negative peaks adjacent to the ex-anode and ex-cathode electrodes for both short (2 hour) and long time (26 hour) DC poling; data is represented with symbols and the model fitting with the lines. The continuous line is a fit of the decay of the UA26h peak next to the anode to just the deep trap release, whereas the dashed line includes decay from both deep and shallow traps for this peak.

Figure 6 shows the change in time of the modulus of space charge density $\left(\rho(t)\right.$ in $\left.\mathrm{Cm}^{-3}\right)$ during decay, separately for the charge peaks near the anode and cathode, see Figure 3, from which it should be noted that the bulk space charge stored in the sample (i.e excluding the induced charge on the electrodes) has a negative polarity for both short and long time DC poling. The fit of this data to the de-trapping model is shown by the lines in Figure 6, and the parameters derived for the fit are given in Table 2. From Figure 6 and Table 2 it can be deduced that the negative peak adjacent to the ex-cathode electrode holds the most charge in the short time poling sample, while in the long time poling sample the charge is shared between two negative bulk peaks; one adjacent to the ex-anode electrode and one adjacent to the ex-cathode electrode, see Figure 3. It is also clear from Figure 6 that we cannot attribute the two regimes of space charge decay in Figure 5a to differences between the cathode and anode bulk space charge peaks, since the cathode peak alone exhibits both a short term and a long term decay regime, albeit one that is weaker than that found for the anode peak.

The charge in the peak adjacent to the cathode in the UA2h sample resides mostly in traps with a trap depth range of 0.81 to $0.93 \mathrm{eV}$, with a small amount of charge (less than $1 / 3^{\text {rd }}$ ) residing in deeper traps with trap depths below about $1.05 \mathrm{eV}$ ( $\mathrm{t}_{1}$ for this set of traps is greater than $10^{5}$ seconds). Similarly the charge in the negative peak adjacent to the cathode in the UA26h sample resides in traps that cover two ranges of trap depth; one shallow trap range with depths between $0.81 \mathrm{eV}$ and $0.89 \mathrm{eV}$ and a deeper trap range with trap depths from $0.89 \mathrm{eV}$ to about $1.73 \mathrm{eV}$. On the other hand the negative charge in the UA26h sample at the peak adjacent to the anode resides in a continuous range of traps whose trap depth ranges from 0.89 to $1.58 \mathrm{eV}$. Thus there is approximately the same range of deep traps available at both the cathode region and anode region in the UA26h sample. In the UA2h sample we cannot compare the anode and cathode region because of lack of data, however the range of traps available at the cathode cannot be much different from that available at the cathode in the UA26h sample, although we are unable to give a precise estimate of the deepest filled other than that it must be well in excess of $1.05 \mathrm{eV}$, see Table 1 . The differences between the UA2h sample and UA26h sample are therefore not due to differences in the available traps in the two materials, nor are they due to differences in the traps available at the anode and the cathode. This latter possibility anyway seemed unlikely as the samples are cut from the same tape and from the same radial region of the original cable insulation. 
Table 2. Parameters derived from decay of the charge density of individual peaks adjacent to the electrodes of the unaged (UA) material.

\begin{tabular}{c|c|ccc}
\hline Parameters & $\begin{array}{c}\text { UA2h of poling } \\
\text { Shallow traps in } \\
\text { peak at Cathode }\end{array}$ & Peak at Anode & $\begin{array}{c}\text { Shallow traps in } \\
\text { peak at Cathode }\end{array}$ & $\begin{array}{c}\text { Deep traps in } \\
\text { peak at Cathode }\end{array}$ \\
\hline $\mathrm{Q}(0)^{\mathrm{a}}$ Coulombs & $1.09 \times 10^{-7}$ & $1.00 \times 10^{-7}$ & $4.18 \times 10^{-8}$ & $6.23 \times 10^{-8}$ \\
$\mathrm{t}_{1}$-seconds & 6 & 180 & 6 & 180 \\
$\Delta_{\min }-\mathrm{eV}$ & 0.81 & 0.89 & 0.81 & 0.89 \\
$\Delta_{\max }-\mathrm{eV}$ & 0.93 & 1.58 & 0.88 & 1.73 \\
$\mathrm{Q}(0) \mathrm{kT} /\left[\Delta_{\max }-\Delta_{\min }\right]$ & $2.35 \times 10^{-8}$ & $3.76 \times 10^{-9}$ & $1.62 \times 10^{-8}$ & $1.91 \times 10^{-9}$ \\
$\mathrm{t}_{2}$-seconds & 618 & $7.23 \times 10^{13}$ & 80 & $2.42 \times 10^{16}$ \\
$\begin{array}{c}\mathrm{N} \text {-number of deep } \\
\text { trap states in } \\
\text { stressed volume per } \\
\text { eV energy interval }\end{array}$ & $5.68 \times 10^{12}$ & $9.08 \times 10^{11}$ & $3.91 \times 10^{12}$ & $4.62 \times 10^{11}$ \\
\hline
\end{tabular}

${ }^{\mathrm{a}}$ The total charge is obtained by summing the charge stored in the deep and shallow traps.

${ }^{\mathrm{b}}$ The concentration of filled traps per $\mathrm{eV}\left(\mathrm{m}^{-3}(\mathrm{eV})^{-1}\right)$ is shown in brackets.

\section{Discussion}

A major difference between the charge in the UA26h sample and that in the UA2h sample is that about $60 \%$ of the charge that formerly resided in the shallow traps at the cathode in the UA2h sample has been transferred to the deep traps, i.e. at the cathode $\mathrm{Q}_{\text {shallow }}(2)=\mathrm{Q}_{\text {shallow }}(26)+\mathrm{Q}_{\text {deep }}(26)$. A possible explanation is the charge system near the cathode was not in thermal equilibrium by two hours of poling and that after 26 hours of poling charges have been transferred from the shallow trap range to the deep trap range as the charge distribution in the traps approached the Boltzmann (Fermi) distribution. However the estimated trap depth range given in Table 2 suggests an alternative explanation. In the UA2h negative cathode peak the shallow trap depth range lies between $0.81 \mathrm{eV}$ and $0.93 \mathrm{eV}$ and the occupied deep trap range lies below about $1.05 \mathrm{eV}$, whereas the cathode peak shallow trap range in the UA26h sample lies between $0.81 \mathrm{eV}$ and $0.875 \mathrm{eV}$, with the filled deep trap range starting at $0.89 \mathrm{eV}$ running to deeper trap energies. It is therefore possible that the extra period of poling has converted some shallow traps to deep traps. If this explanation applied however, we would have to assume that the same range of deep traps were generated near the anode during the extra poling even though there is little (or no) negative charge there to begin with. Though possible this seems unlikely. Also the extra poling period is rather small though the poling field is quite high (50 $\mathrm{kV} / \mathrm{mm}$ ). Thus the more likely explanation is that negative charges that resided at the bottom of the shallow trap range $\mathrm{eV}$ have transferred to the deeper trap range which has become filled up to $0.89 \mathrm{eV}$. In this latter case we must regard the two distributions of traps as arising from different centres or species, with transfer of charge between the two types of trap being slow. The possibility that a long period of poling has converted some shallow traps to deep traps cannot however be fully ruled out without further work.

The negative peak near to the anode exists only in the UA26h and consists of charge that resides entirely in the deep traps. It is tempting to explain the development of this peak to negative charge that has transferred from the cathode to the anode, especially as this explanation would be consistent with a 
system that is not in thermodynamic equilibrium after 2 hours of poling [27], i.e. a larger fraction of charge in the shallower traps than that expected of the Boltzmann population would allow an easier transport of that charge until it reached the counter electrode (i.e. it is more mobile), where a delay in extraction would allow it time to fill the deep traps [17] according to the Boltzmann (Fermi) distribution. However, the equality of the total negative charge in the cathode peak after 26 hours poling with that in the shallow traps after 2 hours poling, and the equality of the negative charge in the cathode peak with that in the negative peak at the anode after 26 hours poling implies that one should be cautious about making such a deduction. Possibly what has happened is that the negative charge at the cathode that is shallow trapped at 2 hours of poling has transferred from the bottom of the shallow trap range to deep traps in the same locality, while the negative charge in the top of the shallow trap range has transited the sample to form the negative peak near the anode. At the same time further negative charge injection from the cathode has taken place, with the charge being trapped into the shallow traps, but not yet reaching a thermal equilibrium (i.e. a Boltzmann distribution). This suggests that the system is in a dynamic equilibrium near the cathode rather than a thermodynamic equilibrium, as in a dynamic equilibrium it does not matter what energy states the charge occupies as long as the charge density remains the same over time. This would be consistent with the observed trapped charge distribution at the cathode after 26 hours poling. The amount of negative charge at the anode has changed during the extra poling period so the existence of a dynamic equilibrium there cannot be demonstrated. Since all the negative charge in this region has reached deep traps this charge may be in thermodynamic equilibrium, however the equality of the total negative charge there with the amount at the cathode indicates that even here the system may be in dynamic equilibrium with the rate of charge arrival equally the rate of extraction. Only longer time experiments would be able to evaluate this possibility which have not yet been carried out.

The analysis given here implies that the negative space charge at the cathode does not exhibit a Boltzmann (Fermi) distribution between the various energy ranges of trap states, i.e. the negative carriers are not in thermodynamic equilibrium. This contention is in keeping with the approach adopted in [29], which showed that the I-V characteristic typical of insulating polymers could be derived from a simulation model in which Schottky injection was combined with trapping, detrapping, and recombination processes to yield a steady state whose currents, field distribution, mobile and trapped charge concentrations were determined by the balance of the various rate processes, i.e. the DC steady state is a dynamic rather than thermodynamic equilibrium. If substantiated these results move the study of space charge distributions and their effect on steady state currents away from the analytical regime available to a thermodynamic equilibrium described for example in [30] to one in which the relative magnitudes of a range of different rate processes have to be considered. The solution for this kind of system can only be obtained by numerical simulation and will have different detailed features for different materials and conditions.

\section{Conclusions}

Our data has shown that poling XLPE peeling for twenty six hours allows time for negative injected space charge from the cathode to transit to the anode, which does not occur to a significant extent in a poling time of two hours. We have also shown that the injected charge is distributed between trap states in two different energy ranges, for charge trapped both near to the cathode and near to the anode. In addition we have shown that the negative charge that is trapped close to the cathode is mostly trapped in shallow states after two hours but is partly transferred to deep traps after 26 hours poling with a consequent longer decay time when the sample was short-circuited. The negative charge that has transferred across the sample to the anode during poling has been delayed in extraction giving a heterocharge peak where the trapped charge has come into thermal equilibrium with the deep traps, with no charge residing in shallow traps. The results are consistent with a charge injection, trapping, and transport system that is not in thermal equilibrium, but instead is in dynamic equilibrium, though the possibility of the conversion of shallow traps to deep traps at the cathode cannot be completely ruled out. 


\section{References}

[1] Mazzanti G, Montanari G C and Dissado L A 2005 Electrical aging and life models: the role of space charge Dielectrics and Electrical Insulation, IEEE Transactions on 12 876-90

[2] Crine J P 2005 On the interpretation of some electrical aging and relaxation phenomena in solid dielectrics Dielectrics and Electrical Insulation, IEEE Transactions on 12 1089-107

[3] Mazzanti G, Montanari G C and Alison J M 2003 A space-charge based method for the estimation of apparent mobility and trap depth as markers for insulation degradationtheoretical basis and experimental validation Dielectrics and Electrical Insulation IEEE Transactions on 10 187-97

[4] Mazzanti G, Montanari G C and Palmieri F 2003 Quantities extracted from space-charge measurements as markers for insulation aging Dielectrics and Electrical Insulation, IEEE Transactions on 10 198-203

[5] Montanari G C, Fabiani D, Bencivenni L, Garros B and Audry C 1999 Space-charge and conduction-current measurements for the evaluation of aging of insulating materials for DC applications Annual Report Conf. on Electrical Insulation and Dielectric Phenomena, CEIDP (Austin, TX, USA: IEEE) 38-42

[6] Montanari G C, Fabiani D, Melloni M and Palmieri F 2002 Diagnostic markers for AC power cable insulation aging based on space charge measurements. Conf. Record of the 2002 IEEE Int. Symp. on Electrical Insulation (Boston, MA, USA: IEEE) pp 464-7

[7] Montanari G C, Mazzanti G, Palmieri F, Motori A, Perego G and Serra S 2001 Space-charge trapping and conduction in LDPE, HDPE and XLPE Journal of Physics D: Applied Physics 34 2902-11

[8] Fothergill J C, Montanari G C, Stevens G C, Laurent C, Teyssedre G, Dissado L A, Nilsson U $\mathrm{H}$ and Platbrood G 2003 Electrical, microstructural, physical and chemical characterization of HV XLPE cable peelings for an electrical aging diagnostic data base Dielectrics and Electrical Insulation IEEE Transactions on, 10 514-27

[9] Tzimas A $2008 \mathrm{PhD}$ Thesis Identification of AC electrothermal ageing markers from ARTEMIS cable peeling University of Leicester, Leicester (http://hdl.handle.net/2381/4053 ) p 196

[10] Tzimas A, Fu M and Dissado L A 2005 Characterization of thermally aged XLPE cable peelings through space charge measurements Annual Report Conf. on Electrical Insulation and Dielectric Phenomena (Nashville, TN, USA: IEEE) 30-3

[11] Tzimas A, Fu M, Dissado L A and Hampton R N 2006 Comparison of XLPE cable peelings with different electro-thermal histories using endurance test and residual charge methods Proc. of the IEEE Int. Conf. on Properties and Applications of Dielectric Materials, (Bali, Indonesia: IEEE) 293-6

[12] Tzimas A, Fu M, Dissado L A, Nilsson U H and Campus A 2007 Space charge characterization of XLPE peelings with a cable prehistory: Before and after AC endurance tests Int. Conf. on Solid Dielectrics, ICSD, (Winchester, UK: IEEE) 474-7

[13] Tzimas A, Rowland S M, Dissado L A, Mingli F and Nilsson U H 2009 Effect of long-time electrical and thermal stresses upon the endurance capability of cable insulation material Dielectrics and Electrical Insulation, IEEE Transactions on 16 1436-43

[14] Maeno T, Futami T, Kushibe H, Takada T and Cooke C M 1988 Measurement of spatial charge distribution in thick dielectrics using the pulsed electroacoustic method Electrical Insulation, IEEE Transactions on $\mathbf{2 3}$ 433-9

[15] Lahoud N, Boudou L and Martinez-Vega J 2007 A Multi-dimensional Model to Describe the Ageing Process in Polymers Used for Electrical Insulation Solid Dielectrics ICSD '07. IEEE International Conference on, 79-81

[16] Dissado L A and Le Roy S 2007 The effect of Contact Charge upon the Injection Current at an Electrode-Insulator Interface Solid Dielectrics ICSD '07. IEEE Int. Conf. on, 31-4 
[17] Le Roy S, Teyssedre G, Laurent C, Dissado L A and Montanari G C 2007 Relative Importance of Trapping and Extraction in the Simulation of Space Charge Distribution in Polymeric Insulators under DC Potentials Solid Dielectrics ICSD '07. IEEE Int. Conf. on, 494-7

[18] Dissado L A, Griseri V, Peasgood W, Cooper E S, Fukunaga K and Fothergill J C 2006 Decay of space charge in a glassy epoxy resin following voltage removal Dielectrics and Electrical Insulation, IEEE Transactions on 13 903-16

[19] Dissado L A, Fothergill J C, See A, Stevens G C, Markey L, Laurent C, Teyssedre G, Nilsson U H, Platbrood G and Montanari G C 2000 Characterizing HV XLPE cables by electrical, chemical and microstructural measurements on cable peeling: effects of surface roughness, thermal treatment and peeling location Annual Report Conf. on Electrical Insulation and Dielectric Phenomena (Victoria, BC, Canada: IEEE) 136-40

[20] Fu M and Chen G 2003 Space charge measurement in polymer insulated power cables using flat ground electrode PEA system Science, Measurement and Technology, IEE Proceedings 150 89-96

[21] Suzuoki Y, Muto H, Mizutani T, Ieda M 1985 Effects of Space Charge on Electrical Conduction in High-Density Polyethylene, J.Phys.D.:Appl.Phys., 18, 2293-2302

[22] Mitsumoto S, Fu M, Dissado L A, Fothergill J C 2006 Short time interval decay measurement of space charge in epoxy resin, IEEJ Trans. FM, Vol .126, No.4, pp.260 - 261

[23] Morin P, Lewiner J, Alquie C, Ditchi T 1998 Study of space charge dynamics in solid dielectrics by simultaneous measurement of external current and space charge distribution, in Space Charge in Solid Dielectrics (Leicester U.K., The Dielectrics Society ISBN0 9533538 $\mathrm{X})$

[24] Dissado L A, Paris O, Ditchi T, Alquie C and Lewiner J 1999 Space charge injection and extraction in high divergent fields Electrical Insulation and Dielectric Phenomena, Annual Report IEEE Conf. on, 23-6

[25] Watson P K 1995 The transport and trapping of electrons in polymers Annual Report Conf. on Electrical Insulation and Dielectric Phenomena (Virginia Beach, VA, USA: IEEE) 21-7

[26] Mizutani T, Suzuoki Y, and Ieda M, 1977, Thermally Stimulated Currents in Polyethylene and Ethyl-Vinyl-Acetate Copolymers, J.Appl.Phys. 48,2408-2414.

[27] Kao K C and Hwang W 1981 Int. Series in Science of the Solid State, 14 (Oxford U.K.:Pergamon press)

[28] Abramowitz M and Stegun I A 1965 Handbook of Mathematical functions (New York: Dover Press)

[29] Baudoin F, Roy S L, Teyssedre G and Laurent C 2008 Bipolar charge transport model with trapping and recombination: an analysis of the current versus applied electric field characteristic in steady state conditions Journal of Physics D: Applied Physics 41025306 (10pp)

[30] Rose A 1955 Space-Charge-Limited Currents in Solids Physical Review 971538 